\title{
Pheochromocytoma: A Rare Cause of Childhood Hypertensive Encephalopathy
}

\author{
ISLAM S ${ }^{1}, \mathrm{~S} \mathrm{KABIR}^{2}$, MORSHED AKMA ${ }^{3}$, ALAM SMS $^{4}$, SIDDIKI MJR $^{5}$
}

\section{Introduction:}

The term pheochromocytoma (in Greek, phios means dusky, chroma means color, and cytoma means tumor) refers to the color of the tumor cells acquired when stained with chromium salts. Roux performed the first surgical resection of a pheochromocytoma in Lausanne, Switzerland in 1926. Later the same year, Charles Mayo performed the first surgical resection in the United States ${ }^{1-2}$.

Pheochromocytoma, a rare tumor found in children and adults, is a catecholamine-secreting tumor. It arises from the chromaffin cells of the sympathetic nervous system (adrenal medulla and sympathetic chain); however, the tumor may develop anywhere in the body. Tumors have been reported to have been found in the neck, mediastinum, retro-peritoneal and pelvic sites and virtually every other site of the body ${ }^{3,4}$. Common locations for extra-adrenal pheochromocytomas include the organ of Zuckerkandl (close to origin of the inferior mesenteric artery), bladder wall, heart, mediastinum, carotid and glomus jugular bodies ${ }^{1-2,5-6}$.

In children, pheochromocytoma is frequently associated with other familial syndromes, such as neurofibromatosis, von Hippel-Lindau disease, tuberous sclerosis, and Sturge-Weber syndrome, and as a component of multiple endocrine neoplasia (MEN) syndromes (MEN 2A, MEN 2B) 3-5. Pheochromocytoma may be inherited as an autosomal dominant trait. Several genes (SDHD, SDHB, SDHC) that belong to the mitochondrial complex II have been identified as involved in the so-called pheochromocytoma-paraganglioma syndrome ${ }^{3,5}$.

1. Dr. Shahnoor Islam, Associate Professor, Department of Pediatric Surgery, Dhaka Medical College Hospital, Dhaka.

2. Shahreen Kabir, HMO, Department of Pediatric Haematology and Oncology, Dhaka Medical College Hospital, Dhaka

3. Dr. AKM Amirul Morshed, Associate Professor, Department of Pediatric Haematology and Oncology, Dhaka Medical College Hospital, Dhaka

4. Dr. S.M. Shafiqul Alam, Consultant, Department of Anaesthesia, Dhaka Medical College Hospital, Dhaka

5. Dr. Jillur Rahman Siddiki, HMO, Department of Pediatric Haematology and Oncology, Dhaka Medical College Hospital, Dhaka

Correspondence: Shahnoor Islam, e-mail-shahnoor6989 @yahoo.com
The biosynthesis and storage of catecholamines in chromaffin cell tumors may differ from the normal medulla although they are functionally and morphologically similar ${ }^{3}$. Catecholamines typically secreted, either intermittently or continuously, include norepinephrine and epinephrine; rarely, dopamine is secreted. Most pheochromocytomas secretes norepinephrine predominantly, where as secretions from normal adrenal medulla are composed of $85 \%$ epinephrine ${ }^{1-3}$.

Patients with pheochromocytomas usually have a thin body habitus. Hypertension may be present in both arms and legs. During a paroxysm, the blood pressure may range from 180-260 mm Hg systolic and from 120-210 mm Hg diastolic. It may cause hypertensive encephalopathy. Upon cardiovascular examination, tachycardia with forceful heartbeat is often found and is easily palpable. Postural hypotension may be present. Patients may feel warm and have pallor of the face and chest. Body perspiration and cool, moist hands and feet may also be found. A mass may be palpable in the neck or in the abdomen on deep palpation. Deep palpation of the abdomen may produce a typical paroxysm. Hypertensive retinopathy and cardiomyopathy are often present. Ophthalmoscopic examination may reveal papilledema, hemorrhages, exudates, and arterial constriction ${ }^{3}$. It is known that catecholamines are metabolized to metanephrines (norepinephrine to normetanephrine and epinephrine to metanephrine) ${ }^{5}$. Free plasma metanephrine levels have been found to be a highly sensitive (100\%) and specific (96.7\%) measure, yielding a negative predictive value of $100 \%{ }^{3}$. Therefore, measurements of fractionated metanephrines in urine or plasma provide superior diagnostic sensitivity over measurement of the parent catecholamines.

Tumor localization is done by either computed tomography (CT) or magnetic resonance imaging $(\mathrm{MRI})^{3,5}$. A well-known method of tumor localization is MIBG, although not performed in Bangladesh. MIBG is a guanethidine analogue resembling norepinephrine and therefore is concentrated by sympatho-adrenergic tissues especially chromaffin tissue of the adrenal 
medulla. In patients where 123I-MIBG scanning is negative, positron emission tomography or octreotide scanning may be useful. The important differentials include Neuroblastoma and Coarctation of aorta ${ }^{3}$.

The definitive treatment of pheochromocytoma is surgical excision of the tumor ${ }^{7-9}$. Transabdominal surgery has been the traditional approach; it allows early ligation of the adrenal vein to minimize systemic catecholamine release during manipulation and also facilitates exploration of the sympathetic chain for multifocality ${ }^{3}$. Alternatively, a laparoscopic adrenalectomy, which has been shown to be a useful technique in patients with tumors smaller than $7 \mathrm{~cm}$ and a body mass index of less than $45 \mathrm{~kg} / \mathrm{m}^{2}$, can be considered $^{3,10}$. The contraindications to laparoscopy include evidence of soft-tissue or vascular extraadrenal extension ${ }^{3}$.

Surgery is usually planned only when appropriate control of blood pressure is achieved. Careful preoperative preparation requires combined alpha and beta blockade to control blood pressure and to prevent intraoperative hypertensive crisis $^{3}$. Alpha-adrenergic blockade, particularly Phenoxybenzamine, is most commonly used $3,5,10$. Alternatives to phenoxybenzamine include Prazosin, Terazosin and Doxazosin $^{3,5}$. Additional beta blocker is required if tachycardia or arrhythmias develop; this should not be introduced until the patient is alpha blocked because unopposed alpha adrenergic receptor stimulation can precipitate hypertensive crisis $^{3,5,10}$. Treatment of a hypertensive crisis due to pheochromocytoma should be based on administration of phentolamine, usually given as an i.v. bolus of $2.5 \mathrm{mg}$ to $5 \mathrm{mg}$ at $1 \mathrm{mg} / \mathrm{min}$ and can be repeated every 5 minutes until hypertension is adequately controlled. Alternatively, control of blood pressure may be achieved by a continuous infusion of sodium nitroprusside at 0.5 to $10.0 \mathrm{~g} / \mathrm{kg}$ per minute ${ }^{5}$. Although a few patients remain hypertensive in the immediate post-operative period, most require treatment for hypotension, which is best remedied by administration of fluids. Hypoglycemia in the period immediately after tumor removal is another problem that is best prevented by infusion of $5 \%$ dextrose started immediately after tumor removal and continuing for several hours thereafter. Post-operative hypoglycemia is transient, whereas low blood pressure and orthostatic hypotension may persist for up to a day or more after surgery and require care with assumption of sitting or upright posture ${ }^{5}$.
The long-term prognosis of patients after operation for pheochromocytoma is excellent, although nearly $50 \%$ may remain hypertensive after surgery. Biochemical testing should be repeated after about 14-28 days from surgery in order to check for remaining disease. On long-term follow-up about $17 \%$ of tumors recur, with about half of these showing signs of malignancy ${ }^{5}$.

\section{Case Report:}

A 10-year-old boy was presented with paroxysmal attacks of severe palpitation, headache and sweating along with chest and abdominal pain for 2 months. His mother also complained that he often suffered from fear of impending death for the same duration. These distressing episodes occurred about 3-4 times per day even when he was at rest, although more pronounced with activity such as bending over or picking up things from the floor. There was no history of significant weight loss, diarrhoea, high colour urine or scanty micturition. He was treated for a single episode of generalized tonic-clonic convulsion as a result of hypertensive encephalopathy before being referred to the department of Paediatrics, Dhaka Medical College Hospital. On examination, the boy was restless, not pale, with a pulse of 140 per minute of regular beat and good volume. Blood pressure was $160 / 100 \mathrm{~mm} \mathrm{Hg}$ which was much above the $95^{\text {th }}$ percentile for his age. His hands and fingers were found to be moist although fine tremor was absent. The boy was also found to be malnourished (Body mass index-12.5) despite his good appetite. Apex beat was found to be on the lateral side of the left nipple, thrusting in nature but heart sounds were normal. There was diffuse tenderness over the whole abdomen with no palpable mass appreciated.Ophthalmologic examination was normal. Other systemic examinations revealed normal findings.

His complete blood count revealed mild anaemia (Hb10.2g/dl), otherwise normal total and differentials. Thyroid hormone, renal function, liver functions, random blood sugar was within normal limit. Twenty four hrs Urinary VMA(Vanillylmandelic Acid) was elevated (13.78mg/24 hrs). Abdominal ultrasonography revealed right sided suprarenal mass $(4.3 \times 4.3) \mathrm{cm}$ with biliary sludge. Computed tomography of abdomen showed "almost rounded ring enhancing mixed density mass lesion with central necrosis measuring $4.3 \times 3.7 \mathrm{~cm}$ was seen in the right suprarenal area and multiple cholelithiasis". No vascular enhancement or displacement was noted. 
Haemolytic cause of cholelithiasis was excluded by a normal hemoglobin electrophoresis. Electrocardiography showed tachycardia and echocardiography was commented as mild concentric left ventricular hypertrophy with otherwise normal cardiac function. X-ray chest showed cardiomegaly. Then the patient was transferred to pediatric surgery department for surgical management.

The first step of the treatment was the reduction of blood pressure towards the normal for his age. Oral Nifedipin SR (20mg) and beta-blocker was started at first, but blood pressure was not yet to be controlled. Cardiac and anaesthetic help were sought. Thereafter, with the addition of Captopril and alphablocker it was brought to normal for his age in a period of 10 days.

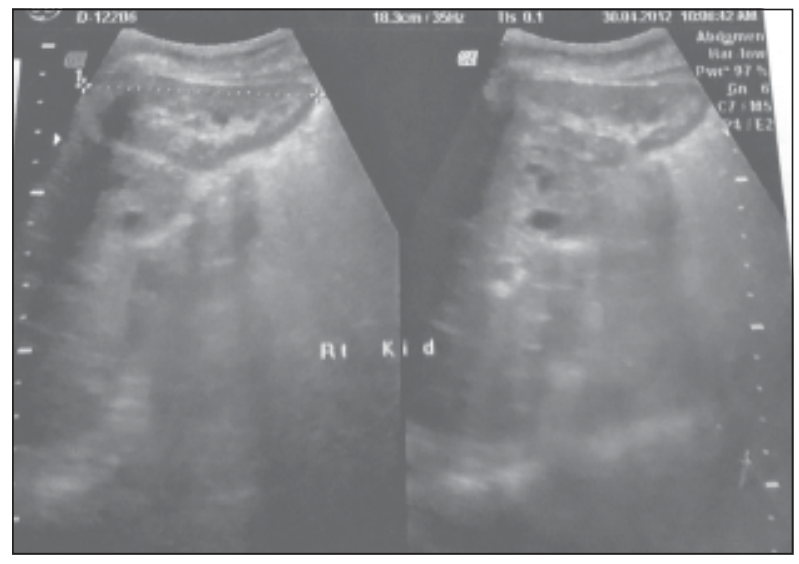

Fig.-1: Ultrasound study demonstrates a right

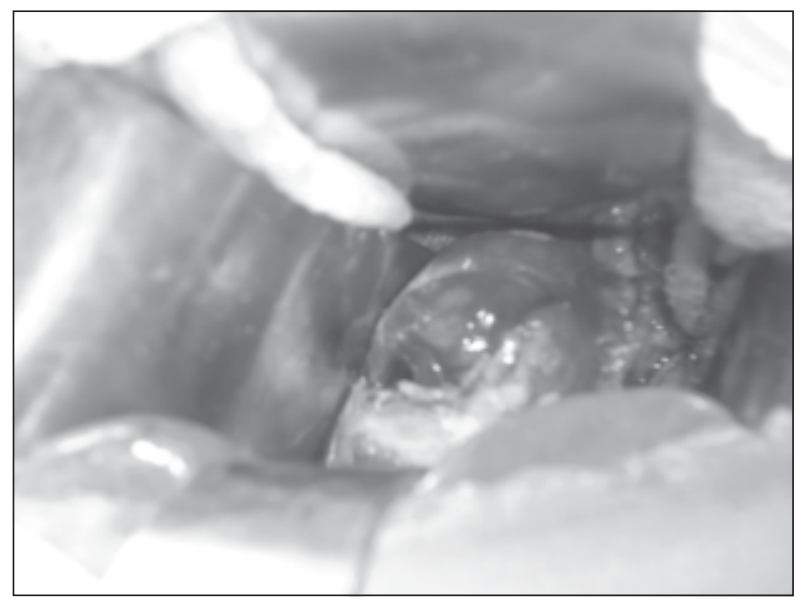

Open right adrenalectomy was done by right subcostal incision. After double ligation of the right adrenal vein the gland was dissected from the surrounding structures and removed. Per-operative cardiovascular events included single episode of hypertension $(130 / 90 \mathrm{~mm} \mathrm{Hg})$ which was combated with Sodium Nitroprusside (50mg) and also a lone episode of hypotension was managed by intravenous ephedrine and fluids. Following surgery patient became normotensive and further antihypertensive was not required. He was discharged on $10^{\text {th }}$ post-operative day with advice for follow up after two weeks. The specimen was sent for histopathological evaluation.

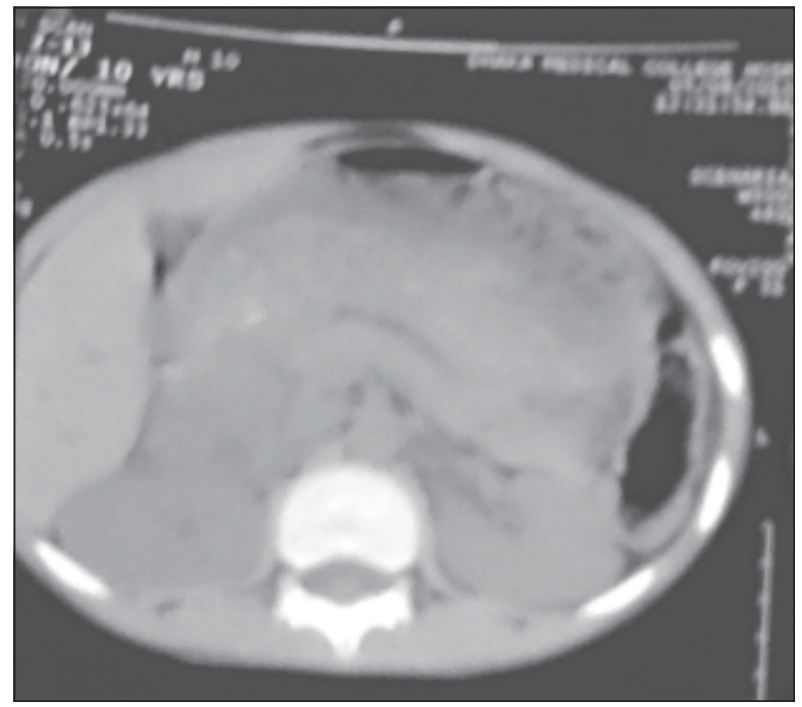

Fig.-2:Computed tomographic scan sided pheocromocytoma Demonstrates a right Sided phe ocromocytoma

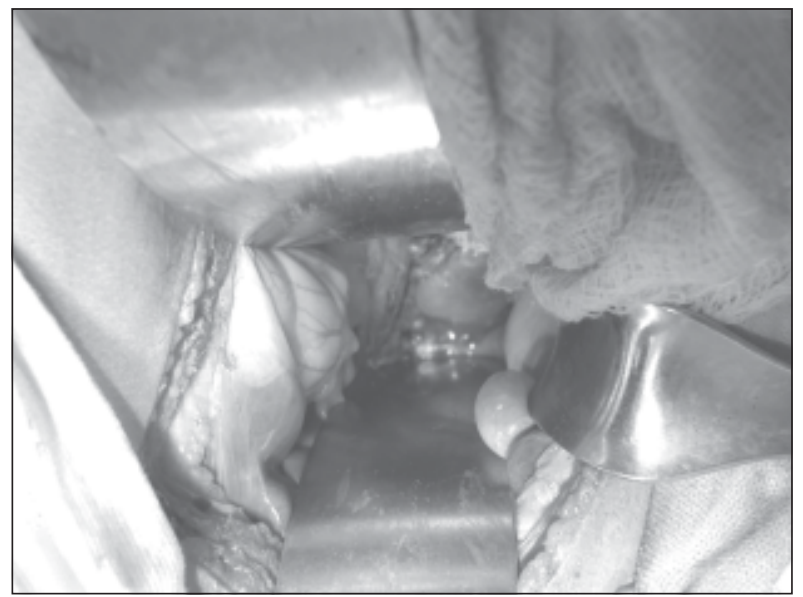

Fig.-5:During operation, a. Before b.after removal of right-sided pheochromocytoma. 


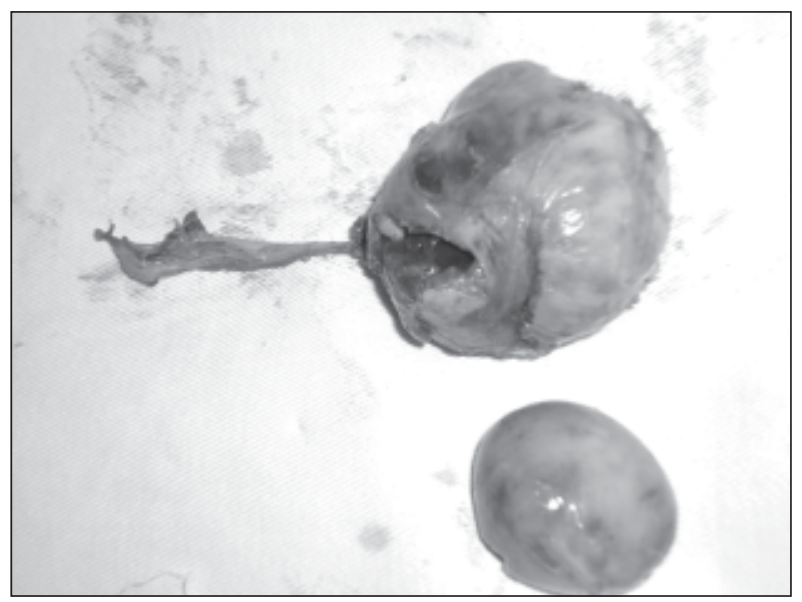

Fig.-5: After resection of pheochromocytoma

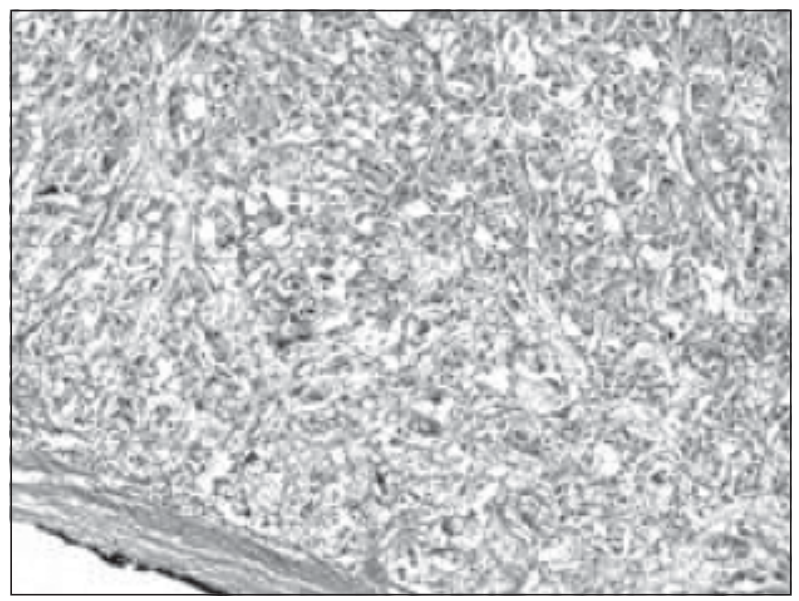

Fig-6: Histological features of pheochromocytoma

Macroscopically, the specimen was measuring about $4.5 \times 4.0 \times 3.0 \mathrm{~cm}$, consisting of grey brown globular tissue. The cut surface was grey brown with some cystic spaces containing dark brown material. Microscopically, the tumour was made of organoid clusters of cells having large nuclei surrounded by abundant cytoplasm. The tumour cells were arranged in trabecular pattern. Capsular invasion was not seen. The report was concordant with our clinical and imaging judgment: Pheochromocytoma .

After more than 9 months of follow up, the blood pressure was in normal ranges without antihypertensive treatment.

\section{Discussion:}

A pheochromocytoma is a neuroendocrine tumor of the medulla of the adrenal glands or extra-adrenal chromaffin tissue that fails to involute after birth. In the fifth week of fetal development, neuroblastic cells migrate from the thoracic neural crest to form the sympathetic chains and preaortic ganglia. These cells are believed to be the precursors of neuroblastomas and ganglioneuromas. Chromaffin cells migrate a second time to the adrenal medulla and also settle near the sympathetic ganglia, the vagus nerve, paraganglia, and carotid arteries which explains the wide ranges of anatomical sites the tumour can be found ${ }^{3}$.

Familial cases are often bilateral or multicentric within an individual adrenal gland. Adrenal pheochromocytomas are most often found on the right side and are sporadic, unilateral, and intra-adrenal. Approximately $6-10 \%$ of the tumors are malignant. The reported incidence rate of pheochromocytomas is approximately 1 case per 100,000 persons, with $10-20 \%$ of cases occurring in children or adolescents. Children have a higher frequency of bilateral tumors than adults and a lower incidence of malignancy ${ }^{3-4,7-}$ 8 . In our patient, the tumour was found on the right side.

The excess catecholamine is responsible for the classical set of sign symptoms of episodic headache, palpitation, sweating, pallor almost always accompanied by hypertension. Others include: chest and abdominal pain, nausea, tremor, anxiety, flushing, dyspnea, weakness, convulsions, etc ${ }^{1,3-5}$. In contrast to adults, in whom there is a high incidence of paroxysmal hypertension, most children have sustained hypertension. The paroxysms are occasionally precipitated by excitement or a particular physical activity, such as bending over or lifting a heavy object. Convulsions secondary to hypertensive encephalopathy may occur ${ }^{3,4}$. Because of its great variety of presentations, pheochromocytoma is often referred as the "Great Imitator" or "Great Mimic" 4,5. Such symptoms we had also in our patient.

In Bangladesh, however, measurement of metanephrine is unavailable and measurement of $24 \mathrm{hr}$ urinary VMA (Vanillylmandelic Acid) is the remaining choice. So, measurement of urinary VMA was done for this case and was elevated.

Tumor localization is done by either computed tomography (CT) or magnetic resonance imaging (MRI) ${ }^{3,5}$. We localized the tumour by computed tomography of abdomen.

In our study, we also performed surgical excision of the tumour by transabdominal route. Careful 
preoperative preparation was done with combined alpha and beta blockade to control blood pressure to prevent intraoperative hypertensive crisis. Per-operative single episode of hypertension $\left(130 / 90 \mathrm{~mm}\right.$ of $\mathrm{Hg},>95^{\text {th }}$ centile) was combated with Sodium Nitroprusside (50mg) and also one episode of hypotension was managed by intravenous ephedrine and fluids. Following surgery patient became normotensive and further anti-hypertensive was not required. Importantly, however, normal postoperative biochemical test results do not exclude remaining microscopic disease so much so that patients should be informed that they are cured and that no further follow-up is necessary.

\section{Conclusions:}

The case is interesting because of the fact, that this is a rare disease in pediatric practice. In the diagnostic plan, we give a high assessment of the complex of image tests, without which the setting of the diagnosis was impossible. The prognosis in our case seems good. Regular follow up of the patient is essential because of the possibility of relapses of the tumour.

\section{References:}

1. Blake MA.Pheochromocytoma Available from http://emedicine.medscape. com/article/124059overview. accessed on 14/11/12

2. Havekes B, Romijn JA,Eisenhofer $G$ et al ;Update on pediatric pheocromocytoma. Pediatric nephrol. $2009 ; 24(5): 943-50$.
3. Vuguin PM, Coppes MJ. Paediatric Pheochromocytoma. Available from http:// emedicine.medscape.com/article/988683overview.accessed on 14/11/12

4. Marinov L. Pheochromocytoma during childhooda case report. Journal of IMAB- Annual Proceeding( Scientific Papers). 2009, book 1, 19-22.

5. Hughes MS, Kebebew E, Pacak K. March2010. Endotext. Available from http:// www.endotext.org/adrenal/adrenal34/adrenalf . accessed on 14/11/12

6. Manger WM, Gifford RW. Pheochromocytoma. J Clin Hypertens. 2002;4(1) :62-72.

7. Khorram MA, Ahlman $\mathrm{H}$, Nilson $\mathrm{O}$ et al. Longterm outcome of a large series of patients surgically treated for Pheochromocytoma. J Intern Med. 2005; 258(1): 55-66.

8. Scholz T, Schulz C, Klose S, Lehnert $\mathrm{H}$. Diagnostic management of benign and malignant pheochromocytoma. Exp Clin Endocrinol Diabetes. 2007; 115(3): 155-9.

9. Hande KR. Adrenal medulla and pheochromocytoma. In: Goldman L, Ausiello D, eds. Cecil Medicine, $23^{\text {rd }}$ ed. Philadelphia, Pa: Saunders, Elsevier; 2007: chap 246.

10. Biswas SK, Alam MJ, Rahman MM. A case report of adrenal Pheochromocytoma. Faridpur Med. Coll. J. 2011; 69(1) : 55-8. 